\title{
Hematoma masivo esofágico con compromiso supraglótico: Reporte de un caso y revisión de la literatura
}

\author{
Massive esophageal hematoma with supraglottic extension: \\ Report of a case and review of the literature
}

\author{
Javiera Pardo J1, Alfredo Abarca A², Katherine Walker J², Carlos Celedón L².
}

\begin{abstract}
RESUMEN
Se presenta el caso de un paciente de 67 años de sexo masculino, hipertenso, diabético y usuario de aspirina en dosis anticoagulante, que consulta en el servicio de urgencia por un cuadro de inicio súbito de dolor retroesternal asociado a disnea severa y estridor. La evaluación mediante nasofibroscopía muestra una gran masa supraglótica que obstruye el $80 \%$ del lumen, compatible con una masa esofágica. La tomografía computarizada de tórax muestra una imagen compatible con un hematoma disecante esofágico de gran extensión, desde laringe hasta la unión gastroesofágica. El paciente se maneja en forma conservadora mediante observación, analgesia y alimentación parenteral. Se evalúa en forma periódica con tomografía computarizada y nasofibroscopía. Evoluciona con reabsorción progresiva del hematoma y disminución del compromiso laríngeo por lo que es dado de alta a los 10 días. Este caso ejemplifica una rara patología que además tiene una forma atípica de presentación, con obstrucción laríngea.

Palabras clave: Hematoma esofágico, compromiso supraglótico, nasofibroscopía, tomografía computarizada.
\end{abstract}

\begin{abstract}
We present a case of a 67 years old hipertense and diabetic male. The patient refered a sudden onset of chest pain associated with severe dysnea and audible stridor. Nasofibroscopy showed a supraglottic mass that obstructed an estimated $80 \%$ of normal lumen, suggestive of an esophageal mass. Thorax CT scan revealed an image of a dissecting esophageal haematoma that compromised most of the esophageal extension, beggining at the laryngeal plane. The patient was managed by conservative means, through observation, analgesia and parenteral nutrition. Close follow up, by CT Scan as well as nasofibroscopy. The patient evolved well, with haematoma reabsorption and decrease of laryngeal obstruction being discharged after 10 days of hospitalization. We report a rare entity that presented in an unusual way.
\end{abstract}

Key words: Esophageal hematoma, supraglottic extension, nasofibroscopy, computed tomography.

Médico Cirujano. Tesista de Magíster en Ciencias Biomédicas, mención Neurociencias, Universidad de Chile.

Médico del Servicio de Otorrinolaringología, Hospital Clínico Universidad de Chile. 


\section{HISTORIA CLÍNICA}

Consulta al servicio de urgencia un paciente de 67 años, sexo masculino, con antecedentes de diabetes mellitus tipo 2, hipertensión arterial y obesidad en tratamiento, además usuario de aspirina 100 $\mathrm{mg} /$ día. Refiere un cuadro de episodio súbito de dificultad respiratoria mientras dormía. El paciente despierta con estridor inspiratorio y dolor retroesternal urente, con imposibilidad para hablar y tragar saliva, esto asociado a tos y náuseas.

En el servicio de urgencia se evalúa permeabilidad de vía aérea y se descarta infarto al miocardio mediante electrocardiograma. Se interpreta como cuadro de origen gastrointestinal y se deja tratamiento sintomático. Sin embargo, el paciente evoluciona con alivio parcial del dolor y persistencia de la disnea, por lo que vuelve a consultar al servicio de urgencia. Ingresa hemodinámicamente estable, con dificultad respiratoria severa y estridor audible. El examen físico general es normal. Es evaluado por otorrinolaringólogo. Al examen destacan restos hemáticos en cavidad oral. Es evaluado mediante nasofibroscopía, apreciándose una masa violácea pediculada, de base posterior no claramente identificable, que pende sobre supraglotis, obstruyendo el $80 \%$ del lumen, excursiona con la respiración y se asocia a gran retención de secreciones en senos piriformes (Figura 1).

A la historia dirigida el paciente niega disfagia y odinofagia en relación a alimentos durante el día previo. Refiere ingesta de granada la tarde anterior. Sin historia de reflujo gastroesofágico.

Se sospecha el diagnóstico de cuerpo extraño esofágico, es evaluado mediante tomografía computarizada de tórax con contraste, que muestra imagen compatible con un extenso hematoma parietal esofágico submucoso, desde esfínter esofágico superior hasta su unión esofagogástrica, con extensión a laringe a nivel de aritenoides izquierdo (Figuras 2 y 3 ). Dadas las condiciones del paciente, se decide conducta expectante y se hospitaliza en el servicio de intermedio quirúrgico, no siendo necesaria traqueostomía de urgencia.

\section{MANEJO}

El paciente se hospitaliza en intermedio quirúrgico. Se mantiene en posición semisentada, con régimen cero y administración de oxígeno por naricera. Se indica famotidina, analgesia y antieméticos en caso necesario. Se solicita hemograma y pruebas de coagulación, las que son normales, descartándose trastorno de coagulación.

El paciente evoluciona estable, con persistencia del apremio respiratorio. Al día siguiente es controlado con nasofibroscopía, que muestra gran disminución del hematoma, mayor al 50\%, el cual cubre parcialmente la supraglotis a nivel de aritenoides

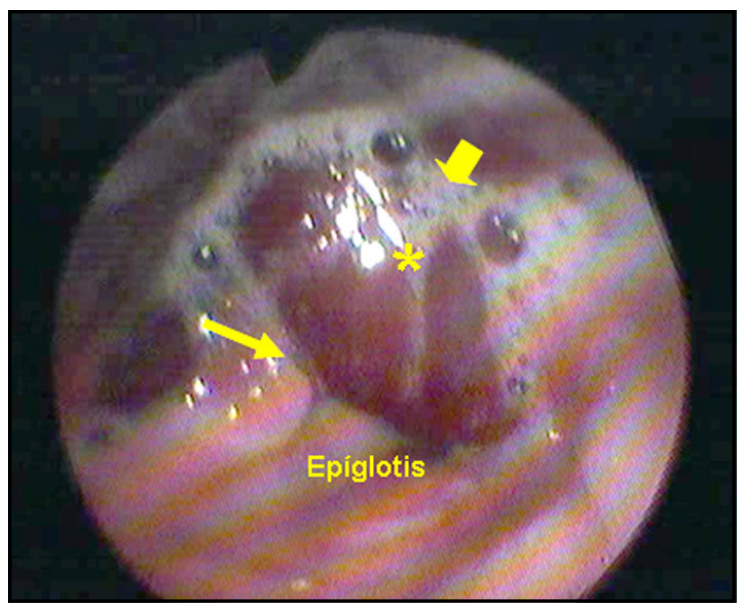

Figura 1. Nasofibroscopía inicial muestra gran masa violácea obstruyendo supraglotis $\left(^{*}\right)$. Flecha muestra aritenoide derecho. Cabeza de flecha muestra retención de secreciones. 

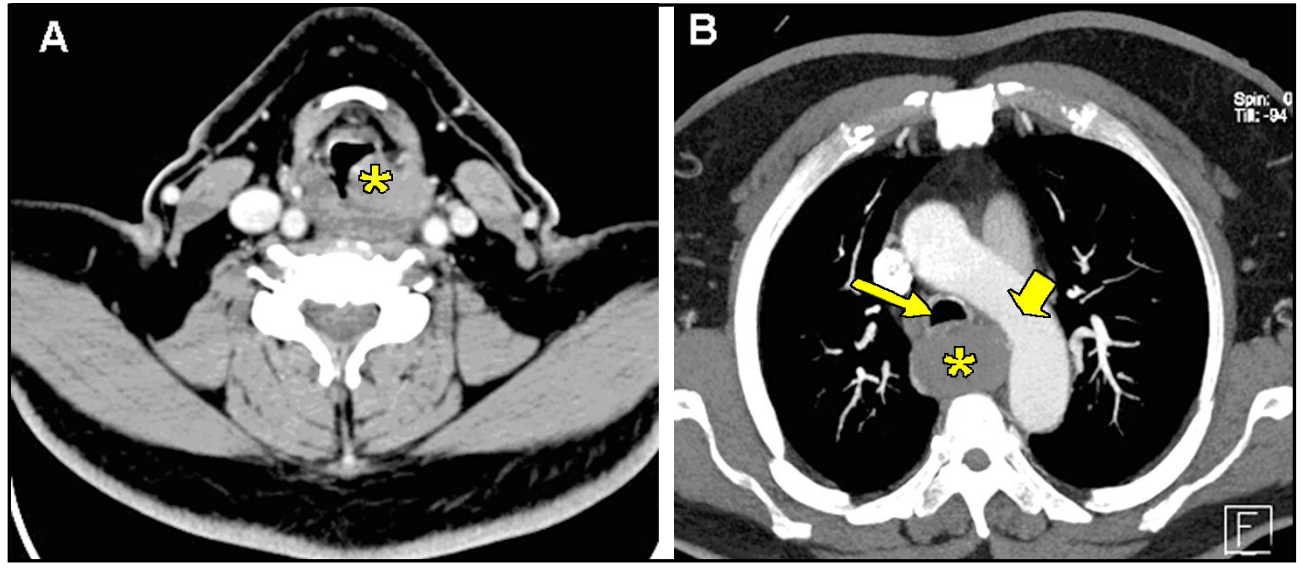

Figura 2. Tomografia computarizada, corte axial. A) A nivel de supraglotis se observa gran hematoma $\left({ }^{*}\right)$ en relación a aritenoide izquierdo, a nivel de esfínter esofágico superior. B) A nivel torácico se observa gran hematoma esofágico submucoso $\left(^{\star}\right)$. Flecha muestra lumen traqueal. Cabeza de flecha muestra arco aórtico.

izquierdo, lográndose visualizar cuerdas vocales y subglotis.

Es evaluado por el equipo de cirugía digestiva, el cual concuerda con el manejo conservador. El paciente es controlado con nasofibroscopía y tomografía computarizada de cuello y tórax con contraste endovenoso cada 24-48 horas. Permanece un total de 10 días hospitalizado, evoluciona favorablemente, con disminución progresiva del apremio respiratorio y disfagia.

La nasofibroscopía de control, previa al alta, muestra gran disminución de hematoma submucoso, con persistencia de equimosis en ambos aritenoides. Cuerdas vocales móviles sin lesiones, espacio glótico conservado con escasa retención de secreciones a nivel de comisura posterior y boca esofágica (Figura 4).

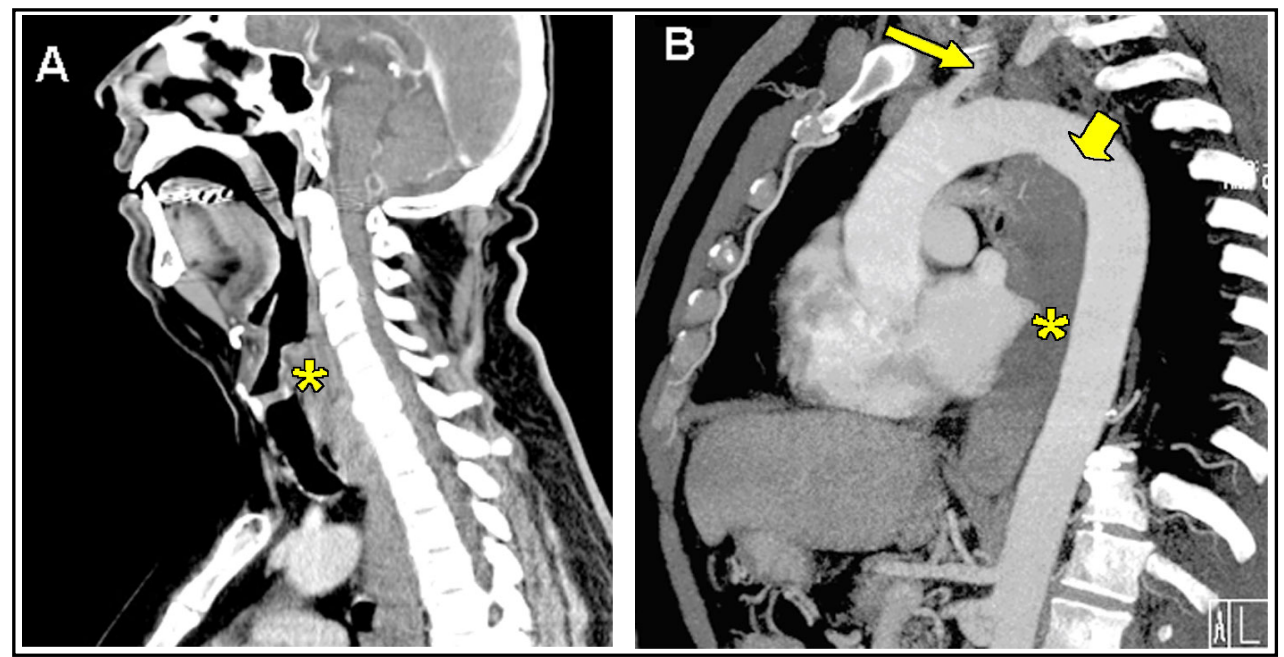

Figura 3. Tomografía computarizada, corte sagital. A) A nivel de supraglotis se observa hematoma (*) solevantado a nivel de esfínter esofágico superior. B) A nivel torácico se observa gran hematoma disecante $\left(^{*}\right)$ que compromete extensamente esófago hasta unión gastroesofágica. Flecha en tráquea. Cabeza de flecha muestra arco aórtico. 


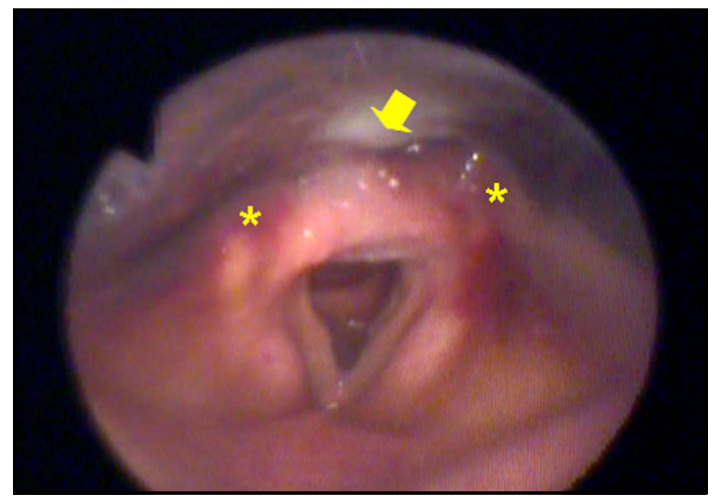

Figura 4. Nasofibroscopía de control, muestra regresión de la lesión, con persistencia de equimosis en relación a ambos aritenoides $\left(^{\star}\right)$. Flecha muestra escasa retención de secreciones.

La tomografía computarizada de control, al décimo día, muestra franca disminución del hematoma esofágico, persistiendo engrosamiento difuso de hipofaringe y segmento torácico (Figura 5).

\section{REVISIÓN DE LA LITERATURA}

\section{Introducción}

El hematoma intramural esofágico (HIE) es una rara patología, incluida dentro del espectro de enfermedades esofágicas como el Mallory- Weiss y el síndrome de Boerhaave ${ }^{1}$, este último corresponde a una ruptura esofágica transmural secundaria a vómitos. Sin embargo, el hematoma esofágico transmural no siempre está asociado a hiperemésis. Otras causas reconocibles son trauma, alteraciones de la coagulación y patología aórtica.

\section{Etiopatogenia}

El hematoma intramural esofágico (HIE) es consecuencia del sangrado de la pared esofágica, que

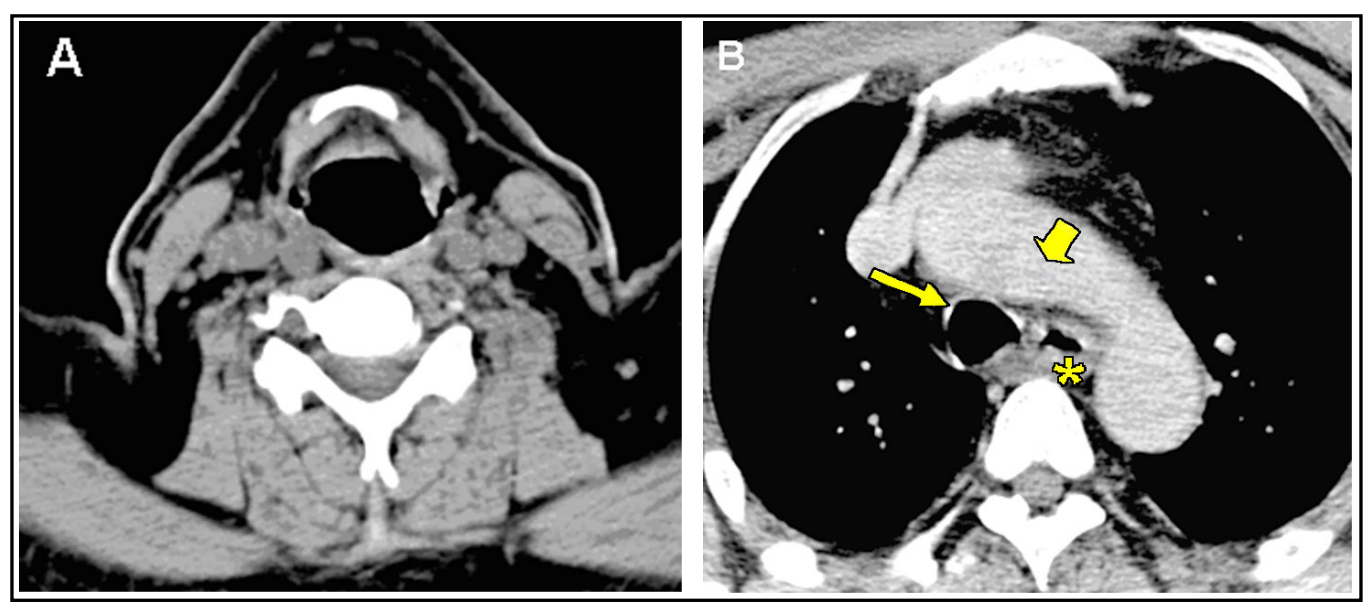

Figura 5. Tomografía computarizada de control, corte axial. A) A nivel de supraglotis se observa disminución del hematoma esofágico. B) A nivel torácico se observa hematoma en vías de reabsorción con parte del lumen esofágico visible $\left(^{\star}\right)$. Flecha en tráquea. Cabeza de flecha muestra arco aórtico. 
usualmente incluye la capa submucosa y ocurre principalmente en la porción distal, la que carece de fibras musculares estriadas, y cuyo sangrado es contenido por estructuras adyacentes como la tráquea y el corazón². El continuo sangrado submucoso genera disección transmural tanto proximal como distal, la que se acumula en forma concéntrica 0 excéntrica en la pared esofágica. En relación a la patogénesis del hematoma intramural esofágico, se distinguen 5 categorías principales: 1) alteración de la hemostasia, 2) secundaria a vómitos, 3) traumática, 4) «espontánea», 5) relacionada a enfermedad aórtica'. Los HIE traumáticos generalmente son iatrogénicos, como complicación de procedimientos endoscópicos, como dilatación esofágica o escleroterapia en el tratamiento de várices esofágicas. Puede ser secundario a traumatismo externo 0 a trauma inducido por la ingesta de alimentos ${ }^{3}$. El concepto de HIE «espontáneo» 0 idiopático se refiere al hematoma que no se asocia a historia de vómitos, alimentación, trauma, alteración de la hemostasia o patología aórtica ${ }^{4}$.

Los HIE asociados a trastornos de la coagulación, ocurren en pacientes que poseen condiciones de base que predisponen el sangrado, como es el caso de pacientes hemofílicos, con leucemia, trombocitopenia, enfermedad renal o por uso de medicamentos que afecten la hemostasia como son los anticoagulantes, antiplaquetarios y agentes trombolíticos, lo que se reporta en más de un tercio de los casos ${ }^{5,6}$.

\section{Patogénesis}

La patogénesis no está del todo clara, sin embargo, existen teorías propuestas de acuerdo a los distintos escenarios clínicos.

Los vómitos causan hipertensión esofágica que generan lesión de la mucosa (síndrome de MalloryWeiss), ruptura transmural (síndrome de Boerhaave) o la formación de un hematoma intramural. En el caso del HIE, la hiperémesis produce aumento de la presión intratorácica, Io que genera una lesión a nivel de la mucosa. Esto produce una hemorragia continua en la pared esofágica y disección progresiva por la acumulación de sangre intramural'1.
Si no existe el antecedente de vómitos, se asume sangrado submucoso con disección transmural, que hasta en un tercio de los pacientes se asocia a trastorno de la coagulación ${ }^{1}$. Se cree que tanto la fuerza emética como algunos mecanismos activos elevan la presión intratorácica lo suficiente como para producir la hemorragia intramural.

Las lesiones traumáticas de alta energía generan rápidos cambios de presión intratorácicos e intraluminales, lo que produciría el sangrado ${ }^{7}$. En relación al trauma por alimentos existen dos teorías: una, es la injuria directa sobre la mucosa y la segunda por aumento de la presión intraesofágica causado por un espasmo esofágico durante su dolor retroesternal ingesta 3 .

En relación a la patología aórtica, se propone la formación de una fístula aortoesofágica. Esta puede ser secundaria a diferentes patologías, como ruptura de un aneurisma aórtico, ruptura de una placa de ateroma, úlcera penetrante, ingesta de un cuerpo extraño o secundario a cáncer esofágico entre otros ${ }^{8}$.

\section{Hallazgos clínicos}

Los hallazgos clínicos no son específicos de esta enfermedad e imitan a una serie de patologías torácicas de origen cardiovascular, pulmonar 0 esofágica. Por lo que el estudio es esencial en dilucidar el diagnóstico.

EI HIE ha sido reportado en pacientes con un amplio rango de edad que va desde los 21 a los 87 años, con una distribución por sexo 2:1, con predominio femenino ${ }^{1,9}$.

Dolor precordial, odinofagia y disfagia son los síntomas más comunes de presentación. El dolor generalmente se localiza en la región retroesternal 0 epigástrica. Por otra parte, la hematemesis es relativamente infrecuente como síntoma inicial y si está presente es de escasa cuantía, requiriendo transfusión sólo en el 10\% de los $\operatorname{casos}^{10}$. La tríada dolor retroesternal, odinofagia/disfagia y hematemesis se presenta sólo en un tercio de los pacientes. Dos de los tres síntomas se describen en $50 \%-80 \%$ de los casos $^{9,11}$. 


\section{Diagnóstico diferencial}

Dentro de las causas de adelgazamiento simétrico o circunferencial de la pared esofágica se incluye el espasmo esofágico difuso, esofagitis y, ocasionalmente, tumores malignos del esófago. Dentro del compromiso asimétrico se encuentran tumores benignos y malignos como carcinoma, leiomiosarcoma y leiomioma'.

\section{Estudio de imágenes: Tomografía computarizada}

La tomografía computarizada es el examen de elección para la evaluación de la pared esofágica, aorta y otras estructuras del mediastino. Es un examen ampliamente disponible, rápido, no invasivo y permite diferenciar patología esofágica de otros procesos torácicos. Permite definir características del hematoma, como compromiso concéntrico 0 excéntrico de la pared, definir bien los límites, que típicamente se extiende hacia la pared posterior, además de definir su extensión $n^{1,12}$.

El protocolo típico de evaluación incluye tomografía con cortes de 3-5 mm. El contraste endovenoso se usa comúnmente. El contraste oral, es utilizado en casos de sospecha de lesión 0 perforación esofágica ${ }^{12}$.

Las masas esofágicas intramurales característicamente no se refuerzan luego de la administración del contraste endovenoso, con excepción de los hematomas secundarios a formación de una fistula aortoesofágica. El hematoma puede comprometer una extensión variable del esófago con grados variables de obliteración del lumen ${ }^{13}$.

En pacientes con coagulación normal, los hematomas generalmente ocurren en esófago distal, probablemente relacionado con la fuerza de cizalla producida durante el vómito. Contrariamente, en pacientes con trastornos de la coagulación, los hematomas se producen frecuentemente en esófago proximal 0 en múltiples focos esofágicos $(60 \%)$. En el periodo hiperagudo, inmediatamente posterior a la hemorragia, el hematoma muestra la misma densidad que la sangre circulante, sin embargo en etapas tardías se torna hipodenso y disminuye de tamaño ${ }^{1}$.

\section{Tratamiento}

En el caso de los hematomas espontáneos el tratamiento es conservador, basado en nutrición parenteral y analgésicos endovenosos. Se observa resolución del hematoma en una a tres semanas, con completa curación de la lesión mucosa y recuperación del tono normal de la pared y de la peristalsis ${ }^{12,14}$. Además, se utilizan inhibidores de los receptores $\mathrm{H} 2$, que disminuyen la producción de ácido gástrico, y antibióticos en caso de fiebre 0 sospecha de infección. El drenaje quirúrgico del hematoma o la reparación de la laceración es raramente requerido ${ }^{1}$.

\section{Discusión}

El hematoma esofágico disecante es una patología rarísima de muy baja incidencia, sin embargo está bien documentada. A nivel internacional, sólo se han descrito 119 casos en 87 publicaciones en lengua inglesa, desde 1968 a la fecha. De éstos, sólo 11 casos $(9,2 \%)$ presentaron sangrado mayor que requirió tranfusión ${ }^{10}$. No existen reportes de hematomas esofágicos disecantes espontáneos con compromiso laríngeo en la literatura. Está descrito un solo caso de obstrucción traqueal por un hematoma esofágico intramural secundario a escleroterapia de várices esofágicas ${ }^{15}$, sin embargo no existen reportes de compromiso respiratorio en hematomas espontáneos.

En relación a este caso, el paciente consulta por la tríada clásica de presentación, consistente en dolor retroesternal, disfagia y hematemesis, típicamente descrita sólo en un tercio de los casos. Tanto el compromiso respiratorio, como su gran extensión, constituyen formas atípicas de presentación. El diagnóstico de hematoma esofágico disecante se clasifica como espontáneo, ya que no existe el antecedente de vómitos previos ni injuria secundaria a ingesta alimenticia. El antecedente de uso de aspirina, antiplaquetario que altera la coagulación, es el principal factor de riesgo para el desarrollo de esta patología en nuestro paciente.

El manejo conservador mediante el uso de analgesia, inhibidores H2 y alimentación parenteral, permitió la correcta resolución del he- 
matoma, la que se evidenció con alivio sintomático de la disnea, disfagia y dolor retroesternal. Se confirmó mediante tomografía computarizada y nasofibroscopía reabsorción significativa del hematoma y mejoría del compromiso laríngeo, a los 10 días de evolución, lo que concuerda con lo descrito en la literatura.

\section{CONCLUSIONES}

El hematoma disecante esofágico es una patología muy poco frecuente, en la que se requiere un alto índice de sospecha ${ }^{16,17}$. El otorrinolaringólogo debe tener presente que esta patología puede presentarse con compromiso laríngeo. La tomografía computarizada con contraste es el examen de elección para hacer el diagnóstico. En este caso, la nasofibroscopía es fundamental para completar el estudio. Su evolución natural es hacia la resolución espontánea en la gran mayoría de los casos, por lo que el tratamiento conservador es una excelente alternativa.

\section{BIBLIOGRAFÍA}

1. Restrepo C, Lemos D, Ocazionez D, Moncada R, GIMÉNEZ C. Intramural hematoma of the esophagus: a pictorial essay. Emerg Radiol 2008; 15: 13-22.

2. Modi P, Edwards A, Fox B, Rahamim J. Dissecting intramural haematoma of the oesophagus. Eur J Cardiothorac Surg 2005; 27: 171-3.

3. KLYGIS LM. Esophageal hematoma and tear from a taco shell impaction. Gastrointest Endosc 1992; 38: 100.

4. Sanaka M, Kuyama Y, Hirama S, Nagayama R, Tanaka $H$, Yamanaka $M$. Spontaneous intramural hematoma localized in the proximal esophagus: truly «spontaneous»? J Clin Gastroenterol 1998; 27: 265-6.

5. Yamashita K, Okuda H, Fukushima MD, Arimura $Y$, Endo T, ImAl K. A case of intramural esophageal hematoma: Complication of anticoagulation with heparin. Gastrointest Endosc 2000; 52: 559-61.

6. Ashman FC, Hill MC, Saba GP, Diaconis JN. Esophageal hematoma associated with thrombocytopenia. Gastrointest Radiol 1978; 3: 115-8.

7. Rozec B, Rigal JC, Leteurnier Y, Lebert C, BLANLOEIL Y. Post-traumatic hematoma of the esophagus. Ann Fr Anesth Reanim 1998; 17: 1160-3.

8. Hollander JE, Quick G. Aortoesophageal fistula: acomprehensive review of the literature. Am J Med 1991; 91: 279-87.

9. Cheung J, Müller N, Weiss A. Spontaneous intramural esophageal hematoma: Case report and review. Can J Gastroenterol 2006; 20(4): 285-6.

10. Shim J, Jang JY, Hwangbo Y, et al. Recurrent massive bleeding due to dissecting intramural hematoma of the esophagus: Treatment with therapeutic angiography. World J Gastroenterol 2009; 15(41): 52325.

11. Enns R, Brown JA, Halparin L. Intramural esophageal hematoma: a diagnostic dilemma. Gastrointest Endosc 2000; 51: 757-9.

12. Chiu Y, Chen J, Hsu C, How C, Hung-Tsang Yen D, Huang C. Spontaneous Esophageal Injury: Esophageal Intramural Hematoma. J Chin Med Assoc 2009; Vol 72: № 9.

13. Picus D, Balfe DM, Koehler Re, Roper CL, OWEN JW. Computed tomography in the staging of esophageal carcinoma. Radiology 1983; 146: 433-8.

14. Abbey P, Sharma R, Garg P K. Spontaneous intramural haematoma of the oesophagus: complete resolution on follow-up magnetic resonance imaging. Singapore Med J 2009; 50(9): e318.

15. Reed AR, Michell WL, Krige Je. Mechanical tracheal obstruction due to an intramural esophageal hematoma following endoscopic variceal sclerotherapy. Am Surg 2001; 67(7): 690-2.

16.Lu M, Liu Y, Liu H, Wu Y, Chu Y, Chu J. 
Spontaneous Intramural Esophageal Hematoma. Ann Thorac Surg 2004; 78: 343-5.
17. Sarah C Thomasset, M.B.Ch.B., David P. Berry. Spontaneous Intramural Esophageal Hematoma. J Gastrointest Surg 2005; 9: 155-6. 